\title{
Negative differential capacitance of quantum dots
}

\author{
S. D. Wang, ${ }^{1}$ Z. Z. Sun,${ }^{1}$ N. Cue,${ }^{1}$ H. Q. Xu, ${ }^{2}$ and X. R. Wang ${ }^{1,3}$ \\ ${ }^{1}$ Physics Department, The Hong Kong University of Science and Technology, Clear Water Bay, Hong Kong SAR, China \\ ${ }^{2}$ Solid State Physics, Lund University, P.O. Box 118, SE-221 00 Lund, Sweden \\ ${ }^{3}$ The International Center of Quantum Structures, CAS, Beijing, China
}

(Received 31 May 2001; published 11 March 2002)

\begin{abstract}
The dependence of charges accumulated on a quantum dot under an external voltage bias is studied. The charge is sensitive to the changes of number of filled levels and the number of conducting levels (channels). We clarify that there are two possible outcomes of applying a bias. (a) The number of conducting channels increases, but the number of filled levels decreases. (b) The number of filled levels increases or does not change while the number of conducting channels (levels) increases with the bias. In case (b), charges are generally expected to increase monotonically with the applied bias. We show, however, that this expectation may not materialize when the electron transmission coefficients depend on bias. Numerical evidences and a theoretical explanation of this negative differential capacitance, i.e., charges accumulated on a quantum dot decrease with applied bias, are presented.
\end{abstract}

DOI: 10.1103/PhysRevB.65.125307

PACS number(s): 73.23.Hk, 73.40.Gk, 73.63.Kv

The capacitance of a quantum dot (QD) has been the subject of many studies. ${ }^{1,2}$ This is largely due to the fact that classical results derived from a macroscopic system are not applicable to a system of nanometer scale at which electron levels are discrete and the density of state is finite. So far, most studies have been on modifications of classical capacitance due to the finite density of states and electron tunneling, such as fluctuation of differential capacitance with respect to the gate voltage. ${ }^{3}$ However the nonlinear chargevoltage characteristics ${ }^{4,5}$ has been less studied.

A QD can be viewed as a potential well which is capable of accommodating electrons. Figure 1 is a schematic diagram of a QD connected to two external leads. The two leads can have different electrochemical potentials, and their difference is called the external bias on the dot. At nonzero bias, a tunneling current may pass through the QD while the electric charges on the QD can also vary with bias. There are three possible ways of applying a bias. Without losing generality, let us assume the electrochemical potential of the left lead $\mu_{L}$ is no less than that of right lead $\mu_{R}$, i.e., $\mu_{L} \geqslant \mu_{R}$. The first way is to raise $\mu_{L}$ while one keeps $\mu_{R}$ unchanged as shown in Fig. 1(b). The dashed line is electrochemical potentials of two leads at zero bias. The second way is to lower $\mu_{R}$, but $\mu_{L}$ does not change. This is shown in Fig. 1(c). The third way is to raise $\mu_{L}$ and to lower $\mu_{R}$ simultaneously as shown in Fig. 1(d). Experimentally, all three ways described in Fig. 1 can be realized easily by controlling the gate voltage of the QD. Here we shall be interested in how the charge $Q(V)$ on the QD change with the bias $V$. Intuitively, the behavior $Q(V)$ depends on how a bias $V$ is applied.

An electron on the dot must occupy on one energy level. At zero temperature, there are three types of energy levels. One is the empty levels on which there are no electrons. They are the levels above $\mu_{L}$. The second type is the filled levels below $\mu_{R}$. The third type is conducting levels (channels) which are between $\mu_{R}$ and $\mu_{L}$, and are partially filled by electrons. In the case of Fig. 1(b), as the external bias increases, the number of conducting channels increases while the number of filled levels does not change. Thus, $Q(V)$ is expected to increase monotonically with $V$ because more and more empty energy levels can accommodate electrons. Similarly, one will expect the charge to decrease with bias in the case of Fig. 1(c). The charge can either increase or decrease with bias in the third case.

In this paper, we shall concentrate on the first case as illustrated in Fig. 1(b). Contrary to the above expectation, we will show that $Q(V)$ can decrease with increasing bias. This behavior will be called negative differential capacitance (NDC) since $d Q(V) / d V<0$.

The electron transport of a quantum dot (QD) at nanometer scale is a many-body problem since the electron-electron
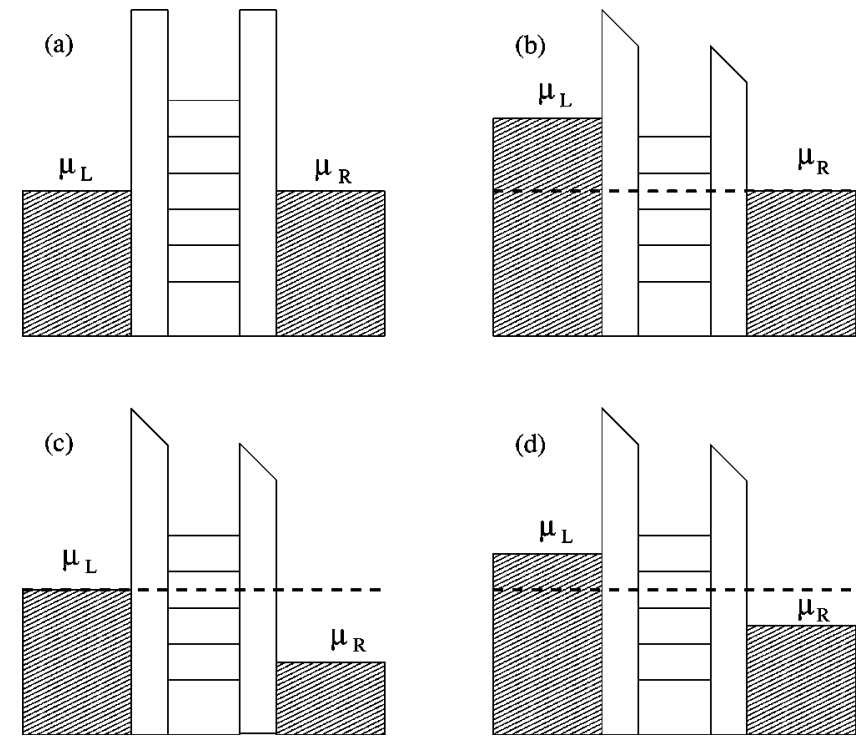

FIG. 1. Schematic diagrams of a quantum dot connected to two external leads. $\mu_{L}$ and $\mu_{R}$ are electrochemical potentials of left and right leads, respectively. The bias applied to the dot is given by $V$ $=\mu_{L}-\mu_{R}$. The dashed lines indicate electrochemical potentials of two leads at zero bias. (a) Zero bias. (b) $\mu_{L}$ is raised while $\mu_{R}$ keeps unchanged. (c) $\mu_{R}$ is lowered while $\mu_{L}$ keeps unchanged. (d) $\mu_{L}$ is raised while $\mu_{R}$ is lowered at the same time. 

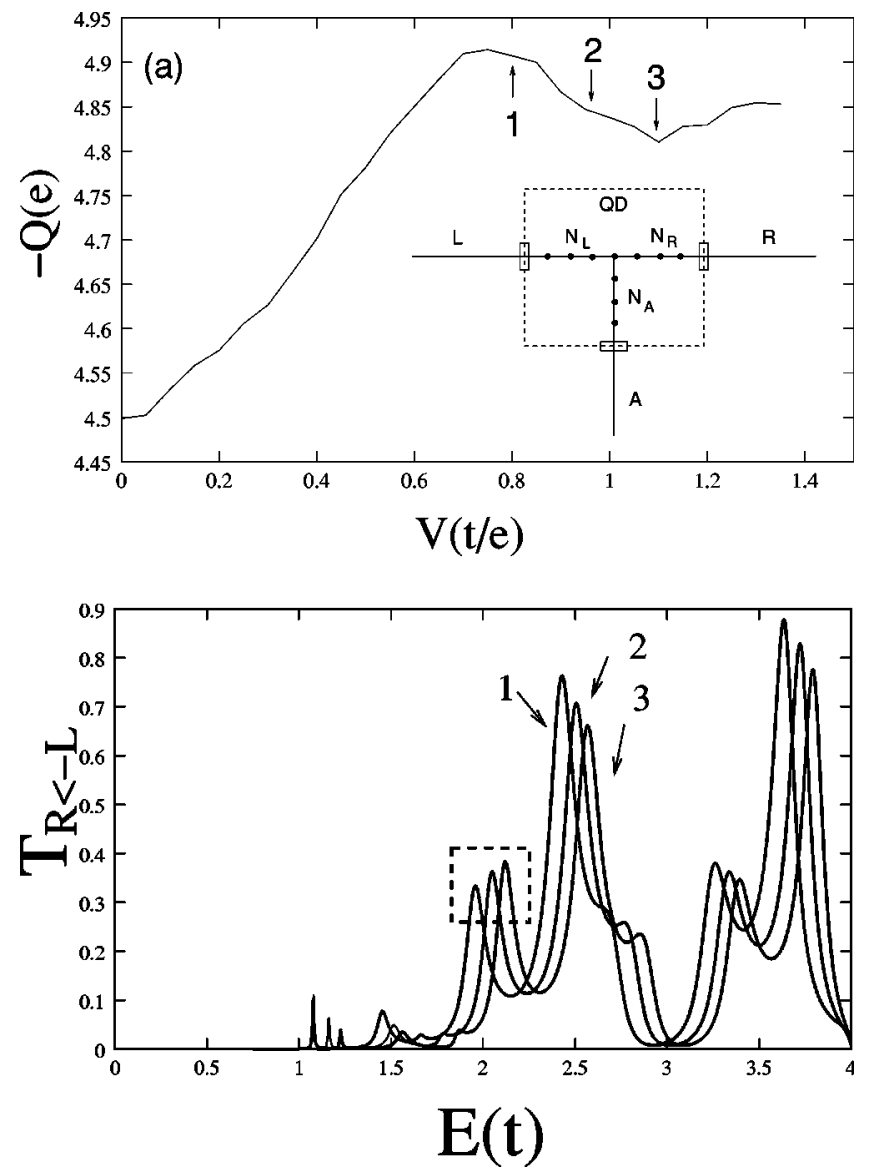

FIG. 2. Numerical results for a quantum dot (QD) of $N_{L}=N_{R}$ $=4, N_{A}=3, U=2 t ; E_{F}=2 t, U_{L}=V, U_{R}=U_{A}=0$, and $b_{L}=b_{R}$ $=b_{A}=4 t$. (a) Charges accumulated in the QD $Q(V)$ vs applied external bias $V . Q(V)$ decreases with bias in the range of $[0.75 t / e, 1.10 t / e]$. Inset of (a): Lattice model of a QD with attached leads. The three joint lines inside the dashed-line box represent the QD. External leads—left $(L)$, right $(R)$, and auxiliary $(A)$-are shown as three lines outside the box. Three small boxes denote potential barriers between leads and the QD. (b) Numerical results of transmission coefficient $T_{L \leftarrow R}$ vs electron energy for bias (1) $0.75 t / e$, (2) $0.95 t / e$, and (3) $1.10 t / e$.

interaction in such a system is usually larger than or comparable to the other energy scales such as the typical level spacing and the thermal energy. It is well known that the Coulomb blockade ${ }^{6-10}$ effect can play an important role in electron transport when the couplings between a QD and external leads are weak. We shall use a self-consistent meanfield theory that has been proved ${ }^{11}$ to be effective in treating the nonlinear electron transport of a correlated system.

Following Ref. 11, a QD connected to two external leads via tunneling barriers is modeled by three joint short lattice wires of length $N_{L}, N_{R}$, and $N_{A}$ as shown in the inset of Fig. 2(a). An auxiliary lead $(A)$ is attached to the wire of length $N_{A}$ in order to include an inelastic process. This auxiliary lead can be regarded as a voltage probe. The cross site is assigned a lattice index $(0,0)$, and the QD spans lattice site $\left(-N_{L}, 0\right), \ldots,\left(N_{R}, 0\right)$ from left to right, and the third wire takes lattice site $(0,1), \ldots,\left(0, N_{A}\right)$. Electron transport occurs between the left $(L)$ and right $(R)$ lead. The Hamiltonian of this system is

$$
\begin{aligned}
\mathcal{H}= & \mathcal{H}_{0}+\sum_{i=-N_{L}}^{N_{R}} U a_{i, 0 ; \uparrow}^{+} a_{i, 0 ; \uparrow} a_{i, 0 ; \downarrow}^{+} a_{i, 0 ; \downarrow} \\
& +\sum_{m=1}^{N_{A}} U a_{0, m ; \uparrow}^{+} a_{0, m ; \uparrow} a_{0, m ; \downarrow}^{+} a_{0, m ; \downarrow},
\end{aligned}
$$

where $\mathcal{H}_{0}$ is the single-particle Hamiltonian of the system. $a_{i, m ; \sigma}$ and $a_{i, m ; \sigma}^{+}$are the annihilation and creation operators, respectively. $\sigma=\uparrow, \downarrow$ is the spin index. The terms containing $U$ describe the electron-electron interactions inside the dot. $\mathcal{H}_{0}$ will be taken as the tight-binding Hamiltonian. On-site energies in the left, the right, and the auxiliary leads are $\varepsilon_{i, 0, \sigma}=U_{L}+2 t$ for $i \leqslant-\left(N_{L}+2\right), \quad \varepsilon_{i, 0, \sigma}=U_{R}+2 t$ for $i$ $\geqslant\left(N_{R}+2\right)$, and $\varepsilon_{0, m, \sigma}=U_{A}+2 t$ for $m \geqslant\left(N_{A}+2\right)$, respectively, with $U_{L}-U_{R}=-e V$, and $t$ being the hopping integral in $\mathcal{H}_{0} . U_{L}, U_{R}$, and $U_{A}$ are the bottoms of conduction bands of the corresponding leads. The electrochemical potentials of left and right leads are $\mu_{L}=E_{F}+U_{L}$ and $\mu_{R}=E_{F}$ $+U_{R}$, where $E_{F}$ is the Fermi energy of the system at equilibrium. The electrochemical potential $\mu_{A}$ of the auxiliary lead will be determined self-consistently. ${ }^{12}$ The potential barriers between the leads and the QD are introduced by assigning $\quad \varepsilon_{-N_{L}-1,0, \sigma}=b_{L}>\mu_{L}, \quad \varepsilon_{N_{R}+1,0, \sigma}=b_{R}>\mu_{R}, \quad$ and $\varepsilon_{0, N_{A}+1, \sigma}=b_{A}>\mu_{A}$. To simulate the case shown in Fig. 1(b), on-site energies on the QD wires are set to be $\varepsilon_{i, m, \sigma}=U_{L}$ $+2 t+\left(N_{L}+1+i\right)\left(U_{L}-U_{R}\right) /\left(N_{L}+N_{R}+2\right)$.

Under the Hartree-Fock approximation, one can obtain an effective single-particle Hamiltonian $\mathcal{H}_{0}^{\text {eff }}$ in which the onsite energies of the QD are $\varepsilon_{m, k ; \sigma}^{\text {eff }}=\varepsilon_{m, k, \sigma}+U\left\langle n_{m, k ; \sigma}\right\rangle$, where $\bar{\sigma}$ is the opposite spin to $\sigma$ and the averaged number of electrons at site $(m, k)$ with spin $\sigma,\left\langle n_{m, k ; \sigma}\right\rangle$, is determined self-consistently by

$$
\left\langle n_{m, k ; \sigma}\right\rangle=-\frac{1}{\pi} \int_{-\infty}^{\mu_{A}} \operatorname{Im}\left\langle m, k ; \sigma\left|G^{\mathrm{eff}}(E)\right| m, k ; \sigma\right\rangle d E,
$$

with the Green's function $G^{\mathrm{eff}}(E)=\left[E-\mathcal{H}^{\mathrm{eff}}+i \eta\right]^{-1}$. The electrochemical potential $\mu_{A}$ can be obtained from the condition that the net current flow into the auxiliary lead is zero. At $T=0$, this is,

$$
I=-\frac{2 e}{h} \int_{\mu_{A}}^{\mu_{L}} T_{A \leftarrow L}(E) d E+\frac{2 e}{h} \int_{\mu_{R}}^{\mu_{A}} T_{R \leftarrow A}(E) d E=0,
$$

where $T_{\beta \leftarrow \alpha}$, which can be calculated by using a transfer matrix formalism, ${ }^{13,14}$ is the transmission coefficients from lead $\alpha$ to lead $\beta$. Then, the charge accumulated inside the QD can be found by

$$
Q=\sum_{\sigma, m=-N_{L}}^{N_{R}} e\left\langle n_{m, 0 ; \sigma}\right\rangle+\sum_{\sigma, m=1}^{N_{A}} e\left\langle n_{0, m ; \sigma}\right\rangle
$$

Below we describe the calculated results for the following settings $N_{L}=4, N_{R}=4, N_{A}=3, E_{F}=2 t, b_{L}=b_{R}=b_{A}=4 t$, $U=2 t, U_{L}=V$, and $U_{R}=U_{A}=0$, where the bias is $V$. The electrochemical potentials of the left and right leads are $\mu_{L}$ $=E_{F}+V=2 t+V$ and $\mu_{R}=E_{F}=2 t$. The bandwidth of all leads is $4 t$. Figure 2(a) is the numerical result of the charge 
vs bias. Here we use $t$ as the unit of energy. We found that the charge increases with bias for a small bias while it decreases for bias in the range of $[0.75 t / e, 1.10 t / e]$. An electron in a QD must stay in an electron level. At zero temperature, there are three types of electron levels. The first type is empty levels whose energies are above the electrochemical potential $\mu_{L}$. The second type is those levels between $\mu_{R}$ and $\mu_{L}$. They are conducting channels partially occupied by electrons. The last type is below $\mu_{R}$. They are fully filled by electrons. In our model, the external bias can modify the potential well of the QD. Thus, it will also change the relative positions of an energy level. Even without going into the detail of a model calculation, one can see two possible ways of decreasing charge on a QD as the bias increases. One is that the number of second and the third type of electron levels decreases as the bias changes. The other is that charge on second type of levels decreases with the bias although the number of such levels does not change.

In order to understand the origin of the negative differential capacitance (NDC) observed in our calculation, we examined the movement of electron levels in the bias range of NDC. Figure 2(b) shows the numerical results of transmission coefficient $T_{R \leftarrow L}$ under bias of $0.75 t / e, 0.95 t / e$, and $1.10 t / e$. The peaks of $T_{R \leftarrow L}$ correspond to the electron levels in QD. In Fig. 2(b), $E=0$ corresponds to the band bottom of the right lead. The absence of peaks below $E=1$ is because, according to our model, no allowed states exist in the left lead in the energy range of $[0 t / e, V t / e]$. At $T=0$, the third type of energy levels, which are fully occupied, are in the range $[0,2 t]$. The conducting channels are the range of $[2 t, V=2 t]$. The electron level corresponding to the peaks inside the dashed-line box of Fig. 2(b) coverts itself from a fully occupied level to a partially occupied conducting channel sometime as the bias increases from $0.75 t / e$ to $0.85 t / e$. This may explain partially the NDC in the range of $[0.75 t / e, 0.85 t / e]$. However, both the numbers of fully occupied levels and partially occupied conducting channels do not change in the NDC region of $[0.85 t / e, 1.10 t / e]$. This shows that the NDC cannot originate from the disappearance of an electron level that can accommodate electrons, but must be due to the second possible mechanism mentioned in the previous paragraph.

We have shown numerically that charges on a QD can decrease with increasing bias when no new electron level leaves or merges with conducting channels. In order to understand the mechanism behind this negative differential capacitance, we consider sequential tunneling of a QD with only one conducting channel of energy $\epsilon\left(\mu_{L}>\epsilon>\mu_{R}\right)$, as schematically illustrated in Fig. 3. Let us assume the electron tunneling rates from the QD to the left lead and the right lead to be $\Gamma_{L}$ and $\Gamma_{R}$, respectively. In the sequential tunneling region, we can ignore the coherent transmission, and the current from the left lead to the dot is ${ }^{15}$

$$
I_{L \rightarrow D}=\frac{2 e}{\hbar} \Gamma_{L} f_{L}(\varepsilon)\left[1-f_{r}(\varepsilon)\right]
$$

Similarly, the current from the dot to the right lead can be written as

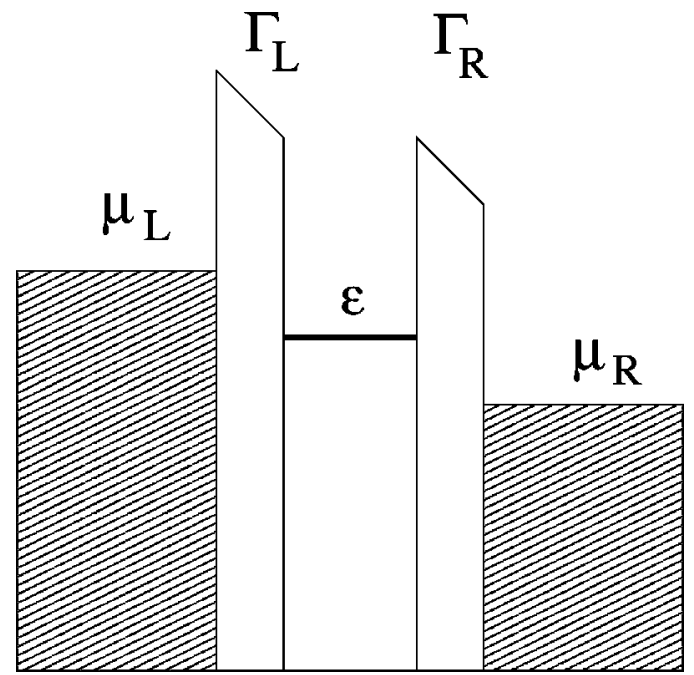

FIG. 3. Schematic diagram of a quantum dot with only one energy level between the electrochemical potentials of two leads. $\varepsilon$ is the energy of the electron state inside the dot. $\mu_{L}$ and $\mu_{R}$ are the electrochemical potentials of the left and right leads, respectively. $\Gamma_{L}$ and $\Gamma_{R}$ are the tunneling rates from dot to the leads.

$$
I_{D \rightarrow R}=\frac{2 e}{\hbar} \Gamma_{R} f_{r}(\varepsilon)\left[1-f_{R}(\varepsilon)\right] .
$$

$f_{L}$ and $f_{R}$ are the Fermi-Dirac distribution of electrons with energy $\epsilon$ in the left and right leads, respectively. $f_{r}$ is the electron occupation probability in the level $\epsilon$. The factor 2 in the above equations accounts for the spin degeneracy. From the continuity of tunneling current, one has

$$
I_{L \rightarrow D}=I_{D \rightarrow R} .
$$

At $T=0$, we have $f_{L}(\varepsilon)=1, f_{R}(\varepsilon)=0$ because $\mu_{L}>\epsilon$ $>\mu_{R}$. Thus, from Eq. (7), the charge in the level is given by

$$
Q=e f_{r}=e \frac{\Gamma_{L}}{\Gamma_{L}+\Gamma_{R}},
$$

which depends on the tunneling rates between the dot and the leads.

In general, an external bias can modify not only the barrier shape, but also can induce level shifts. It is well known that the tunneling rates for a weakly coupled QD can fluctuate wildly from level to level, and with variation in an external parameter such as a magnetic field. ${ }^{16}$ The reason is that the tunneling rates are related to the overlap of wave functions which, in turn, is sensitive to external parameters. One should then expect that a variation in bias can also change the values of the tunneling rates. For a specific level, according to Eq. (8), it is easy to see that the charge accumulated in the level will decrease with increasing bias as long as $\Gamma_{L} / \Gamma_{R}$ decrease with bias. For a real system, many levels may accommodate electrons. The charge in some of these levels may increase with bias while that in the others may decrease. The actual bias dependence of the total charge on the QD should be the average of the two behaviors. Thus, the NDC due to this mechanism is most likely to be found for small systems where only a few levels are important. 
Before ending the paper, we would like to make two remarks. (i) The phenomenon of the negative differential capacitance (NDC) may be important in nanodevice applications. Similar to negative differential resistance, a NDC can also cause instability in electron transport. It may cause the current temporal self-oscillation in superlattices. ${ }^{17}$ (ii) Indeed, the negative differential capacitance discussed here may have already been observed in some recent experiments. $^{18}$
In summary, we have presented numerical evidence of a NDC in a QD. We found that the NDC can occur in a QD as long as not too many levels contribute to electron transport. We show that this NDC can be understood from the bias dependence of the electron tunneling rates in a weakly coupled QD.

We would like to acknowledge the support of the Research Grant Council of HKSAR, China.
${ }^{1}$ T. Christen and M. Buttiker, Phys. Rev. Lett. 77, 143 (1996).

${ }^{2}$ J. Wang, H. Guo, J.-L. Mozos, C. C. Wan, G. Taraschi, and Q. Zheng, Phys. Rev. Lett. 80, 4277 (1998).

${ }^{3}$ A. Kaminski, I. L. Aleiner, and L. I. Glazman, Phys. Rev. Lett. 81, 685 (1998); D. Berman, N. B. Zhitenev, and R. Ashoori, ibid. 82, 161 (1999).

${ }^{4}$ B. Wang, X. Zhao, J. Wang, and H. Guo, Appl. Phys. Lett. 74, 2887 (1999).

${ }^{5}$ M. Krawiec and K. I. Wysokiński, Solid State Commun. 115, 141 (2000).

${ }^{6}$ M. A. Kastner, Phys. Today 46, 24 (1993).

${ }^{7}$ C. W. J. Beenakker, Phys. Rev. B 44, 1646 (1991).

${ }^{8}$ L. W. Lyo and P. Avouris, Science 245, 1369 (1989).

${ }^{9}$ N. S. Wingreen and Y. Meir, Phys. Rev. B 49, 11040 (1994);
Y. Meir, N. Wingreen, and P. A. Lee, Phys. Rev. Lett. 70, 2601 (1993).

${ }^{10}$ C. A. Stafford, Phys. Rev. Lett. 77, 2770 (1996).

${ }^{11}$ H. Q. Xu, Ann. Phys. (N.Y.) 8, 289 (1999).

${ }^{12}$ M. Buttiker, Phys. Rev. B 33, 3020 (1986); Phys. Rev. Lett. 57, 1761 (1986).

${ }^{13}$ W. L. Chan, X. R. Wang, and X. C. Xie, Phys. Rev. B 54, 11213 (1996).

${ }^{14}$ Y. Y. Liu and R. Riklund, Phys. Rev. B 35, 6034 (1987).

${ }^{15}$ S. Datta, Electronic Transport in Mesoscopic Systems (Cambridge University Press, Cambridge, 1995).

${ }^{16}$ R. A. Jalabert, A. D. Stone, and Y. Alhassid, Phys. Rev. Lett. 68, 3468 (1992); A. M. Chang et al., ibid. 76, 1695 (1996).

${ }^{17}$ X. R. Wang and Q. Niu, Phys. Rev. B 59, R12 755 (1999).

${ }^{18}$ A. J. Chiquito, Yu. A. Puscp, S. Mergulhão, J. C. Galzerani, and N. T. Moshegov, Phys. Rev. B 61, 5499 (2000). 